\title{
A Note on Carnap's Result and the Connectives
}

Tristan Grøtvedt Haze

The University of Sydney

\begin{abstract}
Carnap's result about classical proof-theories not ruling out non-normal valuations of propositional logic formulae has seen renewed philosophical interest in recent years. In this note I contribute some considerations which may be helpful in its philosophical assessment. I suggest a vantage point from which to see the way in which classical proof-theories do, at least to a considerable extent, encode the meanings of the connectives (not by determining a range of admissible valuations, but in their own way), and I demonstrate a kind of converse to Carnap's result.
\end{abstract}

Keywords: Connectives, meaning, categoricity, proof-theory, valuation-theory.

\section{Introduction}

In recent years there has been a surge of interest in a previously under-discussed result of Carnap's. ${ }^{1}$ This result, given in Carnap (1943), shows that adding certain non-normal valuations to the set of admissible valuations for propositional logic formulae does not destroy proof-theoretic soundness and completeness. (Exactly what this comes to will be explained shortly.) Carnap's result has been used to cast doubt on the popular and natural idea that the rules of inference of propositional logic encode the meanings of the connectives, and on the idea that the meanings of the connectives can be 'fully formalized'.

\footnotetext{
${ }^{1}$ Since Raatikainen's (2008) reminder of the result, Murzi \& Hjortland have considered how 'intuitionists like Dummett and Prawitz' (p. 480) could respond to this issue, arguing that it does not vitiate their programme. They have also raised but left open the issue of how 'classical inferentialists' (p. 480) might respond. Detailed technical work by Peregrin (2010) has explored 'the power of various inferential frameworks as measured by that of explicitly semantic ones' (Peregrin (2010), p. 255). See also Smith \& Incurvati (2010), Hjortland (2014) and Bonnay \& Westerståhl (2016).

${ }^{2}$ For instance, Raatikainen's gloss of the result is:

It can be shown that no ordinary formalization of logic, and not the standard rules of inference (of the natural deduction) in particular, is sufficient to 'fully formalize' all the essential logical properties of logical constants. That is, they do not exclude the possibility of interpreting logical constants in any other than the ordinary way. (Raatikainen (2008), p. 283.)
} 
In the present note I consider the result, not from the point of view of a proponent or opponent of a philosophical programme, but from a relatively theory-neutral point of view. My aim here is to contribute some considerations which may be helpful in the philosophical assessment of Carnap's result.

In Section 1, I present Carnap's result. In Section 2, I introduce some considerations which show us in a clear light the considerable extent to which ordinary classical proof theories do encode the meanings of the connectives. In Section 3 I show how the simple machinery introduced in Section 2 enables us to demonstrate a kind of converse to Carnap's result. I put this result forward because I find it striking and interesting, and because I have a hunch that it may serve as a corrective to confused or mistaken lines of thought about the philosophical significance of Carnap's result.

\section{Carnap's Result}

In the formal semantics of propositional logic, we assign values to atomic formulae, and the truth-tables determine values for compounds. The resulting complete valuation can be thought of as a total function from formulae to $\{1,0\}-$ a mapping on which every formula is assigned exactly one value. Consider the set of all such total valuation functions $V$. Now, there are obviously other total valuation functions which do not conform to the truth-tables for example, a function on which both some formula and its negation are sent to 1 . Let us call such functions 'non-normal'. We know by soundness and completeness that a formula will be a theorem - where theoremhood is defined proof-theoretically - iff it is assigned 1 by all functions in $V$ (that is, all normal functions). What Carnap showed was that we can add certain non-normal functions to $V$ without destroying soundness and completeness. The most straightforward example is the function $t$ which assigns all formulae to 1 . A formula $\mathrm{F}$ is a theorem iff it is assigned 1 by all functions in the set $V \cup\{t\}$. This holds, because in the case where $\mathrm{F}$ is not a theorem, there will be some function in $V \cup\{t\}$ which does not send $\mathrm{F}$ to 1 , and in the case where $\mathrm{F}$ is a theorem, all functions in $V \cup\{t\}$ will send $\mathrm{F}$ to 1 . In neither case does $t$ get in the way. 


\section{Truth-Tables, Proof-Theories, and What We Can Infer on Their Basis}

One way of looking at the question of the extent to which some proof-theory encodes the meanings of the connectives is to take it for granted that the truth-tables encode their meanings, namely by determining a range of admissible (or normal) valuations, and then ask about the extent to which the proof-theory in question manages to determine the same range. I think it is fair to say that this has been the dominant way of looking at the issue. Here I want to suggest a different perspective: let us consider what may be inferred on the basis of the truth-tables, and then consider, separately, what may be inferred on the basis of an ordinary classical proof-theory. This enables us to see a way in which ordinary classical proof-theories do encode the meanings of the connectives, at least to a considerable extent - not by determining a space of admissible valuations, but in their own way.

Given the statement that $\sim p$ has the value 1 , I can consult the truth-tables and infer that $p$ does not have the value 1 . And vice versa (i.e. given the statement that $p$ does not have the value 1 , I can consult the truth-tables and infer that $\sim p$ has the value 1). From the statement that $p \& q$ has the value 1 , I can infer that $p$ has the value 1 and $q$ has the value 1 , and vice versa. And from the statement that $p \vee q$ has the value 1 , I can infer that $p$ has the value 1 or $q$ has the value 1, and vice versa. Now, a statement which just says, of some formula, that it has the value 1 is not a truth-functional compound. How, then, does it come about that the statement about $\sim p$ is interdeducible with that negative statement about $p$, that the statement about $p \&$ $q$ is interdeducible with that conjunctive statement about $p$ and $q$, and that the statement about $p \vee q$ is interdeducible with that disjunctive statement about $p$ and $q$ ? Obviously, this is due to the truth-tables and their content. This, I think, is a clear vantage point from which to see the way in which the truth-tables encode the meanings of the connectives.

Call a set of PC formulae all of whose members are literals (atoms and negated atoms), and which does not have as members any negations of its other members, a start set. Given the statement that $\sim p$ follows from a start set $\mathrm{S}$, I can consult my chosen proof-theory and infer that $p$ does not follow from $\mathrm{S}$, and vice versa. From the statement that $p \& q$ follows from a start set $\mathrm{S}$, I can infer that $p$ follows from $\mathrm{S}$ and $q$ follows from $\mathrm{S}$, and vice versa. From the statement that $p \mathrm{v} q$ follows from a start set $\mathrm{S}$, I can infer that $p$ follows from $\mathrm{S}$ or $q$ follows 
from S, and vice versa. ${ }^{3}$ Now again, how do these interdeducibilities come about? Obviously, my chosen proof-theory is getting into the act here in a way analogous to the way the truth-tables got into the act in the previous paragraph.

Here we have a vantage point from which to see the way in which ordinary classical proof-theories do, at least to a considerable extent, encode the meanings of the connectives. Granted, there is something funny going on with ' $\sim$ ' in the proof-theory case, and we needed to introduce start sets. Whereas we might be happy to say that the truth-table for ' $\sim$ ' encodes its meaning, it seems wrong to say that our chosen proof-theory encodes the meaning of ' $\sim$ ' all by itself.

\section{A Converse to Carnap's Result}

When we work with valuation-theory, or with proof-theory together with the apparatus of start sets introduced above, we observe the following principles:

The Valuation-theoretic Duality Principle: Every atomic formula is to get exactly one of two values $(0$ or 1$)$. (Then via the truth-tables, for every formula $A$, one of $A$ and $\sim A$ will get the value 1 , the other 0 .)

The Proof-theoretic Duality Principle:The start set S is to contain, for every atom, either that atom or its negation, but not both. (Then via our chosen proof-theory, for every formula A, either A or $\sim \mathrm{A}$ will be derivable from $\mathrm{S}$, but not both.)

The non-normal valuation $t$ which we added to $\mathrm{V}$ in the explanation of Carnap's result breaches the Valuation-theoretic Duality Principle. This can be seen from two angles. One can say that the truth-tables, such as that for ' $\sim$ ', are contravened. Or, one can say that the truth-tables are still in effect, and so formulae will now have more than one value: by

\footnotetext{
${ }^{3}$ The working-out will be easiest in tree systems, followed by natural deduction systems (since they have separate rules for each connective). In an axiom system with few axioms the working-out will be possible in principle but often very involved.
} 
definition of $t$, for any formulae A and $\sim \mathrm{A}$, both will have the value 1, but, via the table for ' $\sim$ ', both with thereby also get the value 0 .

Now: consider the set AS of all start sets, i.e. all sets populated in accordance with the Proof-theoretic Duality Principle. Obviously, a formula can be derived from all sets in AS iff it is a tautology (i.e. gets 1 on all normal valuations). Let us consider the set $\operatorname{AS} \cup\{N\}$, where $\mathrm{N}$ contains all formulae whatsoever. Our converse to Carnap's result can now be stated: a formula A can be derived from all sets in AS $\cup\{N\}$ iff $A$ is a tautology. This holds, because if A is not a tautology, then there will be some member of AS (and thus of AS $U$ $\{\mathrm{N}\}$ ) from which A cannot be derived. If it is a tautology, then of course it will be derivable from $\mathrm{AS}$, and $\mathrm{N}$ does not get in the way, since everything is derivable from N. ${ }^{4}$

\section{References}

Bonnay, Denis \& Westerståhl, Dag (2016). Compositionality Solves Carnap’s Problem. Erkenntnis 81 (4):721-739.

Carnap, Rudolf (1943). Formalization of Logic. Cambridge: Mass., Harvard University Press.

Hjortland, Ole Thomassen (2014). Speech Acts, Categoricity, and the Meanings of Logical Connectives. Notre Dame Journal of Formal Logic 55 (4):445-467.

Murzi, Julien \& Hjortland, Ole Thomassen (2009). Inferentialism and the categoricity problem: Reply to Raatikainen. Analysis 69 (3):480-488.

Peregrin, Jaroslav (2010). Inferentializing Semantics. Journal of Philosophical Logic 39 (3):255 - 274.

Raatikainen, Panu (2008). On rules of inference and the meanings of logical constants. Analysis 68 (300):282-287.

\footnotetext{
${ }^{4}$ Thanks to N.J.J. Smith for encouragement when this result was obtained in the course of work conducted under his supervision in 2010.
} 
Smith, Peter \& Incurvati, Luca (2010). Rejection and valuations. Analysis 70 (1):3 - 10. 